\title{
Midwives' skills in the application of non-pneumatic anti-shock garment for the management of postpartum haemorrhage in Ondo State, Nigeria
}

\section{Christiana Olanrewaju Sowunmi ${ }^{1}$, Olufunke Mercy Iwaola ${ }^{2}$, Gbemisola Bolanle Ogbeye ${ }^{3 *}$}

\author{
${ }^{1}$ Department of Nursing, Babcock University, Ilisan, Ogun State, Nigeria \\ ${ }^{2}$ Department of Nursing, University of Medical Sciences, Ondo, Ondo State, Nigeria \\ ${ }^{3}$ Department of Health Services, Federal University of Technology, Akure, Ondo State, Nigeria
}

Received: 23 September 2018

Accepted: 22 October 2018

\section{*Correspondence: \\ Gbemisola Bolanle Ogbeye, \\ E-mail: gbemisolaogbeye@gmail.com}

Copyright: (C) the author(s), publisher and licensee Medip Academy. This is an open-access article distributed under the terms of the Creative Commons Attribution Non-Commercial License, which permits unrestricted non-commercial use, distribution, and reproduction in any medium, provided the original work is properly cited.

\section{ABSTRACT}

Background: Non-pneumatic Anti-shock garment (NASG) has been identified as a device for effective management of postpartum haemorrhage especially in developing countries where maternal mortality is high. Hence, it is important that midwives are skilled and knowledgeable about its use. This study therefore assessed the midwives' skills in the application of Non-Pneumatic Anti-Shock Garment (NASG) in the management of postpartum hemorrhage.

Methods: This study adopted quasi-experimental research design. The study was done in two secondary health facilities in the state. Sixty-eight midwives participated in the study. Simple random sampling technique was used to select 37 and 31 midwives into experimental and control groups respectively. Written consent was obtained from all the participants. The study involved three stages; Pre-test, an intervention and a post test. Questionnaire and observation rating scale that elicited demographic characteristics, knowledge and skills on the application and removal of NASG was used to collect data pre and post intervention. Data was analyzed using descriptive and inferential statistics.

Results: Mean knowledge score of midwives in the control group in the use of NASG in the management of PPH was found to be $10.32 \pm 4.17$ compared with $12.24 \pm 5.05$ in experimental group pre-intervention. There was a significant increase in knowledge and skills of NASG application, nonetheless the post intervention from experimental group had the highest increase from $6.32 \pm 4.97$ to $17.14 \pm 0.95$.

Conclusions: Educational intervention enhanced skills of midwives in the use of NASG. Therefore, continuous education units in hospitals should include periodic training of midwives on the use of NASG.

Keywords: Non-pneumatic anti shock garment, Nursing intervention, Postpartum haemorrhage, Skills

\section{INTRODUCTION}

Pregnancy and delivery are presumed to be a safe process when well-managed, but a number of times it involves major health risks, even to women with no pre-existing health problem. Thousands of women die all over the world from issues directly associated with pregnancy, delivery and its complications especially, postpartum haemorrhage (PPH). ${ }^{1}$

According to Adesokan, PPH is excessive bleeding from the genital tract after the birth of a baby up to 6 weeks which is in excess of $500 \mathrm{mls}$ or any amount sufficient enough to cause cardiovascular collapse which is 
dangerous to the life of the woman. It is the leading cause of maternal mortality. ${ }^{2}$

Sub-Saharan Africa and South Asia alone accounted for $87 \%$ of global maternal deaths with the situation most dire for women in Sub-Saharan Africa, where one in every 160 women dies of pregnancy related causes during her lifetime, compared with only 1 in 3700 women in developed regions. ${ }^{3}$

As far back as early nineties, Postpartum haemorrhage $(\mathrm{PPH})$ has been the leading cause of maternal death all over the world affecting about $4 \%$ of all pregnancies. ${ }^{4}$ The story has not changed, especially in many developing nations of the world. In 2010, WHO, UNICEF, UNFPA and World Bank estimated that PPH accounted for etiology of $25 \%$ of maternal deaths worldwide. ${ }^{5}$

However, regional variations in the prevalence of maternal death from PPH exist as it could reach as high as $40 \%$ in South East Asia, Latin America and some countries in Africa. ${ }^{6}$ For instance PPH is responsible for about $50 \%$ of maternal mortality in Guatemala and Afghanistan. ${ }^{7}$ Every year, a total number of 529,000 women died due to complications of pregnancy and childbirth. Similarly, statistics has shown that 358,000 women lose their lives due to negative outcome of pregnancy and delivery. ${ }^{8}$

According Reynders et al, majority of these maternal deaths occur within the first few hours after birth. ${ }^{9}$ They occur as a result of either lack or poor management of third stage of labour by skilled midwives or birth attendants. Therefore, the midwives who are the birth attendants need to possess the knowledge and skill in management of third stage of labour, recognize and treat excessive blood loss as well as prevent PPH. Postpartum haemorrhage despite being a leading cause of death among other obstetric complications, it is one of the obstetric complications with established and effective intervention through the use of a low technology device referred to as Non-Pneumatic Anti-Shock Garment (NASG) or life wrap.

NASG is a device in form of a lower bottom suit of an articulated neoprene with Velcro parts which gives lower body circumferential counter pressure that supplies blood to the vital organs of the body, thus reversing hypovolemic shock and decreasing postpartum haemorrhage. Evidence suggests that this promising technology helps overcome further damage and plays a part in sophisticated modern health care units by stabilizing women whilst waiting for definitive haemorrhage therapies such as blood transfusion and surgeries. However, effective utilization of this promising technology requires certain skills that must be possessed by the skilled birth attendant (midwives). ${ }^{7}$

In view of this, WHO in 2013 organized workshops and training on the use of NASG for midwives and nurses in
Nigeria both in the clinical setting and academia to educate them on the availability and the use of the garment in the control of PPH. In addition to this, the Nursing and Midwifery Council Nigeria (NMCN) has incorporated this into basic midwifery curriculum in midwifery training schools. Despite this efforts/training, there is still a gap in the knowledge of application and removal of NASG as evident by submissions of Ogbeye, Ohaeri and Olatubi that though, midwives demonstrated a high level of knowledge about causes and complications of PPH, they have knowledge deficit in its control using anti shock garment, hence, the need for this study. ${ }^{10}$

Objectives of the study seeks to assess the knowledge level of midwives and their skill in the application and removal of NASG; to determine the effect of nursing intervention on knowledge of Midwives in use of NASG in the management of PPH and to determine the effect of nursing intervention in the application and removal of NASG (skills) in the management of PPH.

\section{METHODS}

The study was conducted in two randomly selected secondary health care facilities in Ondo state, Nigeria. Quasi-experimental design was adopted. Ethical approval was sought and obtained from Ondo State Ethical Review Committee with reference details AD.4693Vol. II/42. Entry to the Hospital was through a written letter to the appropriate authority seeking for permission to collect data from the midwives working in labour and post-natal wards of both health institutions. Consent was obtained from all the participants after explaining the procedures to them. Total enumeration method was used to select the sample of thirty-one (31) participants for the control group and thirty-seven (37) participants for the experimental group. The study involved a pre- test, intervention and post-test phase. Two instruments were used for the study; a pre-tested questionnaire and a rating scale. At pre-test, the questionnaires were administered to the participants to assess their baseline level of knowledge on application and removal of NASG. The completed questionnaires were collected back immediately. At intervention, the experimental group was exposed to an educational package in form of lectures and practical demonstration on postpartum haemorrhage, definition, causes, signs and symptoms, anti-shock garment, mode of action, application procedure, removal procedure and contra-indications of application and removal of NASG in four sessions within two weeks, while no intervention was done for the control group. At post intervention, the same questionnaires were administered to both experimental and control groups.

\section{Statistical analysis}

Data collected was analyzed with the aid of Statistical Package for Social Sciences (SPSS) version 21.0. Descriptive statistics such as frequency, mean, mean Gain and Percentages were used to present tables. 
Demographic characteristics, knowledge on anti -shock garment, its application and removal were compared for both experimental and control group. Student t-test was used to evaluate the effect of educational intervention package on knowledge, application and removal of NASG among midwives. The results were accepted to be significant when $\mathrm{P}$ value was less than 0.05 .

\section{RESULTS}

All the study participants were midwives working in the labour and post-natal wards of the selected health facilities. The socio-demographic characteristics of the participants are shown in Table 1.

Table 1: Socio-demographic characteristics of the participants.

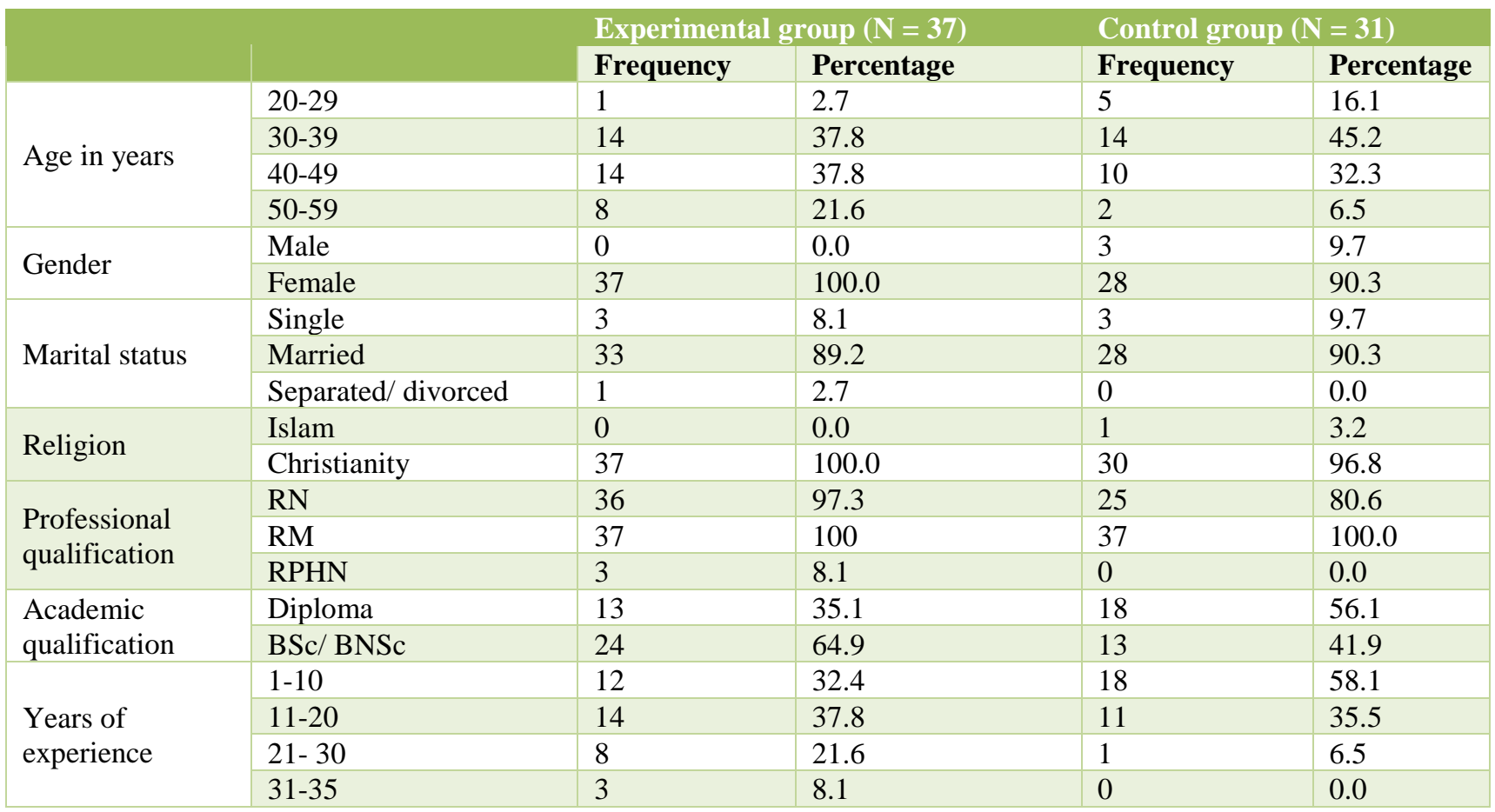

Table 2: Pre and post intervention knowledge level of midwives in the use of NASG in the management of PPH in the control and experimental groups.

\begin{tabular}{|c|c|c|c|c|c|c|c|c|}
\hline \multirow{3}{*}{$\begin{array}{l}\text { Knowledge level } \\
\text { about NASG use }\end{array}$} & \multicolumn{4}{|c|}{ Control } & \multicolumn{4}{|c|}{ Experimental } \\
\hline & \multicolumn{2}{|c|}{ Pre-intervention } & \multicolumn{2}{|c|}{ Post intervention } & \multicolumn{2}{|c|}{ Pre-intervention } & \multicolumn{2}{|c|}{ Post intervention } \\
\hline & $\mathrm{F}$ & $\%$ & $\mathrm{~F}$ & $\%$ & $\mathrm{~F}$ & $\%$ & $\mathrm{~F}$ & $\%$ \\
\hline Low & 16 & 51.6 & 12 & 38.7 & 7 & 18.9 & 0 & 0.0 \\
\hline Moderate & 13 & 41.9 & 15 & 48.4 & 21 & 56.8 & 2 & 5.4 \\
\hline High & 2 & 6.5 & 4 & 12.9 & 9 & 24.3 & 35 & 94.6 \\
\hline Total & 31 & 100.0 & 31 & 100.0 & 37 & 100.0 & 37 & 100.0 \\
\hline Mean & \multicolumn{2}{|c|}{$10.32 \pm 4.17$} & \multicolumn{2}{|c|}{$12.48 \pm 3.79$} & \multicolumn{2}{|c|}{$12.24 \pm 5.05$} & \multicolumn{2}{|c|}{$20.32 \pm 1.61$} \\
\hline Mean gain & \multicolumn{4}{|c|}{2.16} & \multicolumn{4}{|c|}{8.08} \\
\hline
\end{tabular}

The mean number of years of experience was found to be $16.62 \pm 9.15$ (experimental group) and 11.22 \pm 5.24 (the control group). The results also revealed that $24.3 \%$ of the midwives had high knowledge of NASG use in the management of PPH pre-intervention and $94.6 \%$ of the post intervention in the experimental group, whereas $6.5 \%$ of the midwives had high knowledge of NASG use in the management of PPH pre-intervention and $12.9 \%$ of the post intervention in the control group. The mean gain in the knowledge of NASG use in the management of PPH was found to be 2.16 in control and 8.08 in the experimental group (Table 2).

Table 3 revealed that in the experimental study group, $13.5 \%$ of the participants had good skills in NASG application pre-intervention and $94.6 \%$ of the post intervention. While in the control group $9.7 \%$ of the participants had good skills in NASG application pre- 
intervention and $16.1 \%$ of the post intervention. The result also revealed the mean practice (skills) score of $6.32 \pm 4.97$ pre-interventions and $17.14 \pm 4.85$ post intervention in experimental group.

In addition, Table 4 revealed that only $10.8 \%$ and $9.7 \%$ of the participants in the experimental and control groups had good skills respectively in the removal of NASG preintervention compared with $94.6 \%$ and $12.9 \%$ of the participants with good skills post-intervention in experimental and control groups respectively. The mean practice (skills) pre-intervention score was $7.13 \pm 5.74$ and the mean score of $19.13 \pm 1.32$ post intervention in the study group with the mean gain of 12 in experimental and 0.16 in control group.

Furthermore, Table 5 shows the comparison of mean score in the application and removal of NASG in the management of PPH in the experimental group pre and post intervention. There was a statistically significant difference in the participants' skills pre and post intervention $(\mathrm{P}$-value $=0.01)$.

Table 3: Application of NASG by the midwives in the management of PPH pre and post intervention in the control and experimental group.

\begin{tabular}{|c|c|c|c|c|c|c|c|c|}
\hline \multirow{3}{*}{$\begin{array}{l}\text { Practice of NASG } \\
\text { application }\end{array}$} & \multicolumn{4}{|c|}{ Experimental } & \multicolumn{4}{|c|}{ Control } \\
\hline & \multicolumn{2}{|c|}{ Pre-intervention } & \multicolumn{2}{|c|}{ Post Intervention } & \multicolumn{2}{|c|}{ Pre-intervention } & \multicolumn{2}{|c|}{ Post intervention } \\
\hline & $\mathrm{F}$ & $\%$ & $\mathrm{~F}$ & $\%$ & $\mathrm{~F}$ & $\%$ & $\mathrm{~F}$ & $\%$ \\
\hline Poor & 20 & 54.1 & 0 & 0.00 & 12 & 38.7 & 8 & 25.8 \\
\hline Fair & 12 & 32.4 & 2 & 5.4 & 16 & 51.6 & 18 & 58.1 \\
\hline Good & 5 & 13.5 & 35 & 94.6 & 3 & 9.7 & 5 & 16.1 \\
\hline Total & 37 & 100.0 & 37 & 100.0 & 31 & 100.0 & 31 & 100.0 \\
\hline Mean & \multicolumn{2}{|c|}{$6.32 \pm 4.97$} & \multicolumn{2}{|c|}{$17.14 \pm 0.95$} & \multicolumn{2}{|c|}{$7.32 \pm 4.35$} & \multicolumn{2}{|c|}{$7.84 \pm 4.85$} \\
\hline Mean gain & \multicolumn{4}{|c|}{10.82} & \multicolumn{4}{|c|}{0.52} \\
\hline
\end{tabular}

Table 4: Removal of NASG by the midwives in the management of PPH pre and post intervention in the control and experimental group.

\begin{tabular}{|c|c|c|c|c|c|c|c|c|}
\hline \multirow{3}{*}{$\begin{array}{l}\text { Practice of NASG } \\
\text { application }\end{array}$} & \multicolumn{4}{|c|}{ Experimental } & \multicolumn{4}{|c|}{ Control } \\
\hline & \multicolumn{2}{|c|}{ Pre-intervention } & \multicolumn{2}{|c|}{ Post Intervention } & \multicolumn{2}{|c|}{ Pre-intervention } & \multicolumn{2}{|c|}{ Post intervention } \\
\hline & $\mathrm{F}$ & $\%$ & $\mathrm{~F}$ & $\%$ & $\mathrm{~F}$ & $\%$ & $\mathrm{~F}$ & $\%$ \\
\hline Poor & 19 & 51.4 & 0 & 0.00 & 21 & 67.7 & 20 & 64.5 \\
\hline Fair & 14 & 37.8 & 3 & 5.4 & 7 & 22.6 & 7 & 22.6 \\
\hline Good & 4 & 10.8 & 34 & 94.6 & 3 & 9.7 & 4 & 12.9 \\
\hline Total & 37 & 100.0 & 37 & 100.0 & 31 & 100.0 & 31 & 100.0 \\
\hline Mean & \multicolumn{2}{|c|}{$7.13 \pm 5.74$} & \multicolumn{2}{|c|}{$19.13 \pm 1.32$} & \multicolumn{2}{|c|}{$6.68 \pm 6.25$} & \multicolumn{2}{|c|}{$6.52 \pm 5.45$} \\
\hline Mean gain & \multicolumn{4}{|c|}{12} & \multicolumn{4}{|c|}{0.16} \\
\hline
\end{tabular}

Table 5: T-test to compare mean scores in the application and removal of NASG in experimental group pre and post intervention.

\begin{tabular}{|l|l|l|l|l|l|l|l|l|l|}
\hline & Groups & N & Mean & Std. deviation Std. error mean & T value & Df & P value Remark \\
\hline $\begin{array}{l}\text { Practice } \\
\text { of } \\
\text { NASG }\end{array}$ & $\begin{array}{l}\text { Pre- } \\
\text { intervention }\end{array}$ & 37 & 13.54 & 10.61 & 1.74 & -12.95 & 36 & 0.01 & Significant \\
\hline
\end{tabular}

Level of significance:0.05

\section{DISCUSSION}

This study has shown that there was statistically significant difference between the posttest knowledge scores of the experimental and control groups on their knowledge of Anti shock garment in the management of postpartum haemorrhage as well as the skills involved in the application and removal of Anti shock garment.
In the control group, half of the participants had low knowledge of NASG use pre-intervention compared to $38.7 \%$ post intervention. $41.9 \%$ of midwives had moderate knowledge compared to $48.4 \%$ midwives post intervention. This is consistent with the submissions of previous studies in different settings that staff responsible for maternity services in many countries have severe gaps in skills related to prevention of PPH including lack of 
training in Active Management of Third Stage of Labour (AMTSL). ${ }^{11-14}$ In addition, very few of the participants in the control group had high knowledge of NASG use in the management of $\mathrm{PPH}$ and $12.9 \%$ post intervention. Koblinsky et al, earlier established poor knowledge of nurses and midwives in the management of $\mathrm{PPH}$, affirming that not only are many of the techniques for treating the source of PPH available only to physicians at tertiary level facilities (mostly surgical options including compression sutures, ligation of arteries, and hysterectomy), but evidence suggests that nurses and midwives may not have adequate training or may not be able to consistently and correctly use management options available to them (bimanual compression, parenteral uterotonics, IV fluids, and blood transfusions). ${ }^{15}$ The pre and post nursing intervention on knowledge of midwives in the use of NASG in the experimental group showed that midwives in the experimental group had high knowledge of NASG use post intervention compared to the control group. The NASG knowledge among midwives in the experimental group increased from $12.24 \pm 5.05$ pre-intervention to $20.32 \pm 1.61$ post intervention. This is closely related to the submissions of Nazy in a study aimed to evaluate the effect of a medium fidelity simulation based training on nurses' knowledge, performance and clinical judgment of postpartum haemorrhage management that the level of knowledge before program implementation was mainly fair and poor between the experimental and control groups. ${ }^{16}$ Additionally, there was no statically significant difference between the two groups as regards all aspects of knowledge, but after program implementation, there was a statically significant difference between the two groups in favor of the experimental group, progressive improvement in all knowledge aspect was obvious with $80 \%$, reported good knowledge while $64 \%$ of the control group had poor knowledge with a statistically significant difference between the two groups $(\mathrm{P}=0.0001)$. It can therefore be concluded that educational intervention/ training for maternity nurses had a positive effect in improving nurses' knowledge, performance skills and, capture the skills to construct effective clinical judgment in managing postpartum haemorrhage.

Furthermore, Bates et al posited that lack of training in the use of new technologies like NASG among health care workers contribute to high incidence of post-partum haemorraghe among women. ${ }^{17}$ This assertion for the need of training is therefore affirmed by the findings of this study as nurses and midwives exposed to training brought about improved knowledge outcome as well as improved skills in application of NASG.

The skills of the participants in the application of NASG improve post intervention $(6.32 \pm 4.97$ vs $17.14 \pm 0.95)$ compared to control group $(7.32 \pm 4$ vs $7.84 \pm 4.85)$. This result showed that more participants in the experimental group have good practice of NASG application and removal post intervention compared to control group. Also, there was a significant difference in the practice of
NASG application and removal pre and post intervention in the experimental group $(\mathrm{p}=0.01)$. Therefore, findings of present study is consistent with that of Hauswald et al, who found out that educational intervention in form of training yielded significant impact on the health care workers knowledge in the utilization of NASG in the management of PPH. ${ }^{18}$ Also that training on the use of modern techniques in the management of PPH has been shown to be successful in obstetric units. ${ }^{19,20}$

\section{Implication of the study}

This study shows that midwives in Ondo state are aware of the existence of non-pnuematic antishock garment as a device used in the management of postpartum haemorrhage. They however demonstrated knowledge deficit in the application and removal of the garment before intervention, however, this improved significantly after intervention among the experimental group which implies that midwives as skilled attendants have significant role to play in the management and prevention of postpartum haemorrhage which is the leading cause of maternal mortality hence should be exposed to continuous in-service training on current trends in its management in other to improve their knowledge, performance skills and, capture the skills for effective management of postpartum haemorrhage.

Limitations of the study: The study has an inherent limitation. The fact that Anti shock garment is relatively new in the management of postpartum haemorrhage made the process of literature search tedious as there are only very few studies that has been conducted in this area in Nigeria. Most studies have concentrated on its effectiveness in the management of PPH little has been done on the skills of midwives in its use.

Suggestions for further studies: The findings of this study have been able to reveal the need for further studies. Futures studies should therefore be directed towards assessment of the availability and the use of the garment in primary health care facilities; assessment of midwives' proficiency in the use of this garment in the management of postpartum haemorrhage; the study should be replicated in other senatorial district of the state.

\section{CONCLUSION}

Knowledge of midwives in the use of NASG in the management of PPH was observed to be poor preintervention. However, with adequate educational intervention, midwives were found to have good knowledge of NASG in the management of PPH and were able to use NASG effectively. It is, therefore, important that efforts should be directed at regular training of midwives on NASG as the educational intervention program has significantly improved midwives' knowledge and skills in the management of postpartum haemorrhage. 


\section{Recommendations}

Based on the findings from the study, the following recommendations are made:

1. The importance of this garment in the management of postpartum haemorrhage which constitute about $25 \%$ causes of maternal mortality cannot be over emphasized. It is therefore important that this garment should be made available in all health institutions offering maternity services.

2. Up to date training on the use of this garment should be organized from time to time to keep midwives abreast of current practice.

3. All stakeholders, government and non-governmental organizations alike should subsidize the cost of the garment so that private maternity homes can also acquire the garment.

4. Availability of the garment should be made one and important criteria in accrediting maternity homes.

5. Nursing and midwifery council of Nigeria should revise the schools of Nursing and Midwifery curriculum to include contents on NASG use in the management of postpartum haemorrhage.

Funding: No funding sources

Conflict of interest: None declared

Ethical approval: The study was approved by the Institutional Ethics Committee (AD.4693Vol. II/42)

\section{REFERENCES}

1. Maya ET, Buntugu KA, Ako L, Srofenyoh EK. Condom tamponade in the management of primary postpartum haemorrhage: a report of three cases in Ghana. Af J Reprod Heal. 2015;19(3):151-7.

2. Adesokan FOO. Reproductive health for all ages. Faxwell Nigeria Limited, Ekiti State. 2010.

3. World Health Organization. Maternal Mortality. 2014. Fact Sheet. Available at: http://www.who.int/mediacentre/factsheets/fs348/en.

4. Combs CA, Murphy EL, Laros RK Jr. Factors associated with postpartum haemorrhage with vaginal birth. Obstet Gynaecol. 1991;77(1):69-76.

5. World Health Organization, UNICEF, UNFPA \& The World Bank. Trends in maternal mortality: 1990 to 2008: Estimates developed by WHO, UNICEF, UNFPA and The World Bank 2010. Available at: http:// www.who.int/reproductivehealth/publications/monitori ng/ 9789241500265/en/index.html.

6. Miller S, Martin HB, Morris JL. Anti-shock garment in postpartum haemorrhage. Best Pract Res Clinic Obstet Gynaecol. 2008;22(6):1057-74.

7. Ojengbede OA, Morhason-Bello IO, Galadanci H, Meyer C, Nsima D, Camlin C. Assessing the role of the non-pneumatic anti-shock garment in reducing mortality from postpartum hemorrhage in Nigeria. Gynecol Obstet Investig. 2011;71(1):66-72.
8. World Health Organisation Managing Complications in Pregnancy and Childbirth; A guide for Midwives and Doctors. Geneva: World Health Organization. Af J Reprod Heal. 2015;19(3):151-7.

9. Reynders FC, Senten L, Tjalma W, Jacquemyn Y. Postpartum hemorrhage: practical approach to a lifethreatening complication. Clin Exp Obstet Gynecol. 2006;33(2):81-4.

10. Ogbeye GB, Ohaeri BM, Olatubi MI. Midwives knowledge and attitude towards the use of anti-shock garment in the control of post partum haemorrhage in selected hospitals in Ondo State. Int J Heal Sci Res (IJHSR). 2015;5(9):389-95.

11. Cherine M, Khalil K, Hassanein N, Sholkamy H, Breebaart M, Elnoury A. Management of the third stage of labor in an Egyptian teaching hospital. Int J Gynecol Obstet. 2004;87(1):54-8.

12. Oladapo OT, Fawole AO, Loto OM, Adegbola O, Akinola OI, Alao MO, et al. Active management of third stage of labour: a survey of providers' knowledge in southwest Nigeria. Arch Gynecol Obstet. 2009;280(6):945-52.

13. Prevention of Postpartum Hemorrhage Initiative. Tackling the biggest maternal killer: How the Prevention of Postpartum Hemorrhage Initiative strengthened efforts around the world. Washington, DC: PATH. 2009. Available at: http://www.path.org/fi les/MCHN_popphi_tbmk_fi nal_rpt.pdf.

14. Ajenifuja KO, Adepiti CA, Ogunniyi SO. Post partum haemorrhage in a teaching hospital in Nigeria: a 5-year experience. Af Heal Sci. 2010;10(1):71-4.

15. Koblinsky M, Matthews Z, Hussein J, Mavalankar D, Mridha MK, Anwar I, et al. Going to scale with professional skilled care. Lancet. 2006;368(9544):137786.

16. Nazy NH. Effect of a medium fidelity simulation based training on nurses' knowledge, performance and clinical judgment of postpartum hemorrhage management. IOSR J Nurs Heal Sci. 2017;6(2):89-100.

17. Bates I, Chapotera GK, McKew S, Van Den Broek N. Maternal mortality in sub-Saharan Africa: the contribution of ineffective blood transfusion services. BJOG. 2008;115(11):1331-9.

18. Hauswald M, Williamson MR, Baty GM, Kerr NL, Edgar-Mied VL. Use of an improvised pneumatic antishock garment and a non-pneumatic anti-shock garment to control pelvic blood flow. Int $\mathrm{J}$ Emerg Med. 2010;3(3):173-5.

19. Birch L, Jones N, Doyle PM, Green P, McLaughlin A, Champney C, et al. Obstetric skills drills: evaluation of teaching methods. Nurse Edu Tod. 2007;27(8):915-22.

20. Guise JM. Anticipating and responding to obstetric emergencies. Best Pract Res Clinic Obstet Gynaecol. 2007;21(4):625-38.

Cite this article as: Sowunmi CO, Iwaola OM, Ogbeye GB. Midwives' skills in the application of non-pneumatic anti-shock garment for the management of postpartum haemorrhage in Ondo State, Nigeria. Int J Reprod Contracept Obstet Gynecol 2019;8:209-14. 